\section{Embarazos exitosos en hemodiálisis crónica: experiencia de un único centro}

\author{
FRANCISCO ESPINOZA ${ }^{1,3}$, ROMINA ROMEO ${ }^{a}$, MARCELA URSU ${ }^{1}$, \\ AUGUSTO TAPIA ${ }^{2}$, ANTONIO VUKUSICH ${ }^{1,3}$
}

\section{Pregnancy during dialysis. Experience in six patients}

Background: The frequency of pregnancies during dialysis is increasing. This condition requires changes in the dialysis schedule and nutritional approach. Aim: To report the experience in six patients with terminal kidney disease who became pregnant. Material and Methods: Retrospective review of medical records of women with terminal kidney disease in dialysis who became pregnant in a period of 27 years. Results: We recorded six successful pregnancies among women in hemodialysis treatment aged $32 \pm 4$ years. The mean dialysis-time per week was $19.5 \pm 2.7$ hours and $K t / V$ was $1.55 \pm 0.17$. The mean systolic blood pressure was $130 \pm 13.3$ $m m H g$. The mean packed cell volume of the group increased from $22.7 \%$ during pregestational stage to $30.2 \%$ during third trimester of pregnancy. All patients received an intensive treatment for anemia. The most common symptom of pregnancy was hyperemesis. The mean gestational age (GA) at diagnosis was $13.4 \pm 4.7$ weeks. All patients had preterm deliveries at a GA of $33 \pm 1.7$ weeks, and $66 \%$ of offspring were appropriate for gestational age. Conclusions: A multidisciplinary approach allows high rate of successful pregnancies during hemodialysis.

(Rev Med Chile 2013; 141: 1003-1009).

Key words: Pregnancy, highrisk; Renal dialysis; Renal insufficiency.

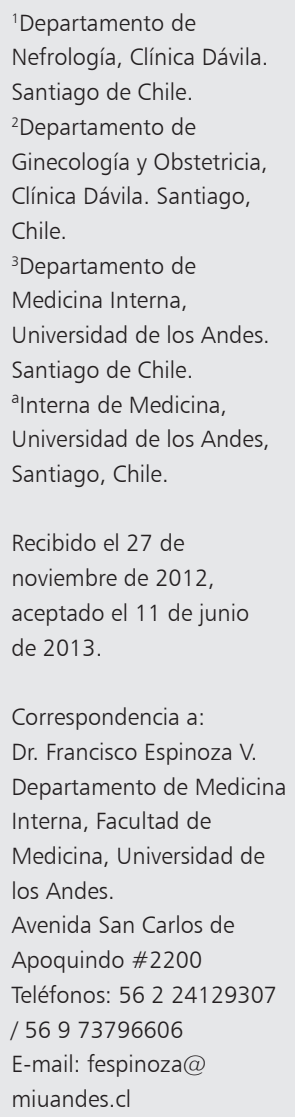

$\mathrm{D}$ esde la publicación del primer caso de un embarazo exitoso en hemodiálisis (HD) (Confortini et al, 1971) ${ }^{1}$, diversas series clínicas coinciden en que esta entidad es aún infrecuente, pero tiene una incidencia creciente y se asocia a un mejor pronóstico materno-fetal.

Datos de la literatura señalan que la tasa de concepción de una mujer en diálisis es de $0,3 \%$ a $1,5 \%{ }^{2}$, la cual es significativamente menor a la de una mujer sana que intenta embarazarse, donde la tasa de concepción es de $45 \%$ por cada ciclo menstrual. Por otra parte, la incidencia de embarazo de pacientes en diálisis está entre 1 y $4 \%{ }^{3,4}$. En cuanto al pronóstico, el registro europeo, a comienzo de la década 1980-89, reportaba una tasa de sobrevida fetal cercana al $40 \%{ }^{5}$. Actualmente, la tasa de éxito es mayor al 70\%, incrementándose entre 10 y $20 \%$ por década ${ }^{6,7}$.

Asistir un embarazo en HD es un enorme desafío. La prescripción dialítica y sus complicaciones habituales deben considerar los cambios fisiológicos que ocurren durante el embarazo. La volemia y el peso seco se incrementan, la hipertensión arterial (HTA) suele agravarse, el trabajo miocárdico aumenta para compensar un estado hiperdinámico, la anemia se profundiza y aumenta el riesgo de desnutrición.

El objetivo del artículo es presentar, por primera vez, una serie chilena de embarazos en $\mathrm{HD}$, que describe los resultados exitosos de todos los casos que hemos atendido en nuestra unidad de HD.

\section{Pacientes y Métodos}

Se realizó una revisión retrospectiva de los embarazos ocurridos en pacientes con enfermedad renal terminal y HD, durante los 27 años de 
actividad de nuestra unidad. El trabajo tiene un diseño descriptivo. Las variables que se registraron fueron: edad, semanas de gestación al diagnóstico de embarazo, causa de la enfermedad renal crónica (ERC), edad gestacional, vía y causa de interrupción del embarazo, peso de nacimiento del recién nacido (RN), hematocrito (Hto) del $\mathrm{RN}$, características de la HD y variables como Hto, presión arterial (PA), albuminemia, calcemia $\left(\mathrm{Ca}^{+2}\right)$, fosfemia (P) y producto $\mathrm{Ca}^{+2} \mathrm{x} P$ de las pacientes durante el embarazo. Para calcular la dosis de diálisis (Kt/V por sesión) se utilizó un modelo bicompartimental de Daurgirdas- $95^{8}$.

\section{Resultados}

\section{Aspectos generales}

En 27 años (1985-2012) se registraron 6 embarazos únicos de mujeres distintas, que requirieron HD durante su gestación. Desconocemos, sin embargo, nuestra tasa de incidencia de embarazos en diálisis. Cinco de los seis casos fueron posteriores al año 2000 (Tabla 1).

El promedio de edad de las pacientes fue de $31,6$ años ( $\mathrm{DE} \pm 3,8)$. Respecto a la etiología de la ERC, hubo una paciente en la cual no se logró establecer la causa (caso 1), una paciente con nefroesclerosis hipertensiva (caso 2), dos pacientes con nefropatía lúpica (casos 3 y 5), una paciente con una glomerulonefritis crónica (caso 4) y una paciente con riñón poliquístico (caso 6).

El caso 2 tenía dos embarazos previos exitosos antes del diagnóstico de la ERC y del ingreso a diálisis.

Cuatro pacientes (66\%) estaban en tratamiento con HD antes de embarazarse. En ellas, el tiempo promedio de permanencia en HD era de 18,8 meses. De las restantes, una paciente inició HD con el diagnóstico de embarazo, dado que la ERC se descubrió en el mismo momento (caso 2). La otra paciente, portadora de una nefropatía lúpica, se había independizado del tratamiento dialítico y mantenía un clearance de creatinina cercano a 20 $\mathrm{ml} / \mathrm{min}$. En ella, se reinició HD con el diagnóstico de embarazo, luego de haber experimentado una descompensación, probablemente por reactivación de su enfermedad de base (caso 3).

\section{Aspectos relacionados a la terapia dialítica}

El promedio de horas de HD semanal en las 4 pacientes que se sometían a la terapia previo al embarazo fue de $10,5 \mathrm{~h}$ y el promedio de $\mathrm{Kt} / \mathrm{V}$ fue de 1,38 (DE $\pm 0,23)$. El promedio de horas de HD semanal de la totalidad del grupo durante el embarazo fue de 19,5 h $(\mathrm{DE} \pm 2,7)$ y el del Kt/V fue de $1,55(\mathrm{DE} \pm 0,17)$. Esto representa un incremento de $185 \%$ en la duración de la HD y de $112 \%$ en el $\mathrm{Kt} / \mathrm{V}$. El promedio de nitrógeno ureico sanguíneo (NUS) prediálisis durante el embarazo fue de 33,9 $\mathrm{mg} / \mathrm{dL}(\mathrm{DE} \pm 9,7)($ Tabla 2).

\section{Tabla 1. Características y resultados materno-fetales de 6 pacientes con ERC tratadas con HD frecuente y prolongada}

\begin{tabular}{|lcccccc|}
\hline & Caso 1 & Caso 2 & Caso 3 & Caso 4 & Caso 5 & Caso 6 \\
\hline Edad (años) & 24 & 33 & 28 & 34 & 33 & 35 \\
Año de embarazo & 1988 & 2001 & 2003 & 2005 & 2008 & 2010 \\
Semana diagnóstico & 7 & 14 & 14 & 12 & 20 & 9 \\
\hline Fórmula obstétrica & GOPOA0 & G2P2A0 & G1P1A1 & G2P2A2 & G2P2A2 & G0P0A0 \\
EG al parto (semanas) & 34 & 34 & 32 & 33 & 33 & 32 \\
Tipo de parto & Cesárea & Cesárea & Cesárea & Cesárea & Cesárea & Cesárea \\
Indicación de interrupción & OA / CIE & PH & CIE & ClE & RCIU & CIE \\
Peso de nacimiento (g) & 2.700 & 2.010 & 2.276 & 1.969 & 1.570 & 1.705 \\
& AEG & AEG & GEG & AEG & PEG & AEG \\
\hline Hto RN (\%) & 50 & 50 & 50 & 52 & 49 & 51 \\
\hline
\end{tabular}

$\mathrm{EG}=$ Edad gestacional. $\mathrm{OA}=$ Oligohidroamnios. $\mathrm{PH}=$ Polihidroamnios. $\mathrm{ClE}=$ Colestasia intrahepática del embarazo. $\mathrm{RCIU}=$ Retardo del Crecimiento Intrauterino. AEG = Adecuado a la edad gestacional. GEG = Grande a la edad gestacional. PEG = Pequeño para la edad gestacional. RN = Recién Nacido. 
Tabla 2. Características de la hemodiálisis y del nitrógeno ureico sanguíneo prediálisis durante el embarazo de 6 pacientes con ERC tratadas con HD frecuente y prolongada

\begin{tabular}{|c|c|c|c|c|c|c|}
\hline & Caso 1 & Caso 2 & Caso 3 & Caso 4 & Caso 5 & Caso 6 \\
\hline Horas de HD semanal ( $\mathrm{x}$ días/sem) & $24(4 \times 6)$ & $21(3,5 \times 6)$ & $17,5(3,5 \times 5)$ & $18(3 \times 6)$ & $18(3 \times 6)$ & $18(3 \times 6)$ \\
\hline Kt/N por sesión & 1,8 & 1,5 & 1,4 & 1,74 & 1,5 & 1,4 \\
\hline Membrana (C/P) & $\mathrm{C} 1,2 \mathrm{~m}^{2}$ & P $1,6 \mathrm{~m}^{2}$ & P $1,6 \mathrm{~m}^{2}$ & P $1,6 \mathrm{~m}^{2}$ & P $1,6 \mathrm{~m}^{2}$ & P $1,8 \mathrm{~m}^{2}$ \\
\hline Flujo sanguíneo (ml/min) & $250-300$ & $300-400$ & $250-300$ & $250-300$ & $250-300$ & $250-300$ \\
\hline Calcio/ $\mathrm{HCO}_{3}(\mathrm{mEq} / \mathrm{lt})$ baño HD & $2,5 / 30$ & $3,5 / 35$ & $3,5 / 35$ & $3,5 / 35$ & $3,5 / 35$ & $3,5 / 35$ \\
\hline $\begin{array}{l}\text { NUS pre-HD (mg/dL) (promedio } \\
\text { trimestres) }\end{array}$ & 23 & 39 & 50 & 26,6 & 30 & 34,5 \\
\hline
\end{tabular}

$\mathrm{HD}=$ Hemodiálisis. $\mathrm{C}=$ Cuprofan. $\mathrm{P}=$ Polisulfona. $\mathrm{HCO} 3=$ bicarbonato. $\mathrm{NUS}=$ Nitrógeno ureico sanguíneo.

Tabla 3. Presión arterial, calcemia, fosfatemia y producto CaxP de 6 pacientes con ERC tratadas con HD frecuente y prolongada

\begin{tabular}{|c|c|c|c|c|c|c|}
\hline & Caso 1 & Caso 2 & Caso 3 & Caso 4 & Caso 5 & Caso 6 \\
\hline $\begin{array}{l}\text { PA promedio durante } \\
\text { el embarazo }(\mathrm{mmHg})\end{array}$ & $122 / 77$ & $150 / 92$ & $121 / 83$ & $134 / 80$ & $110 / 70$ & $140 / 80$ \\
\hline $\begin{array}{l}\text { Tratamiento farmacológico de } \\
\text { la HTA }\end{array}$ & - & $\begin{array}{l}\text { metildopa } \\
1,5 \mathrm{~g} / \mathrm{día}\end{array}$ & - & $\begin{array}{l}\text { hidralazina } \\
150 \text { mg /día }\end{array}$ & - & $\begin{array}{l}\text { nifedipino } \\
20 \mathrm{mg} / \text { día }\end{array}$ \\
\hline $\begin{array}{l}\mathrm{Ca}^{+2} / \mathrm{P} \text { promedio durante } \\
\text { el embarazo }(\mathrm{mg} / \mathrm{dL})\end{array}$ & $8,8 / 2$ & $8,1 / 2,8$ & $8,8 / 2$ & $8,1 / 2$ & $8,1 / 3,3$ & $8,5 / 3,5$ \\
\hline Producto Ca x P & 17,6 & 22,6 & 17,6 & 16 & 27,5 & 29,8 \\
\hline
\end{tabular}

$\mathrm{PA}=$ Presión Arterial. $\mathrm{Ca}^{+2}=$ Calcio. $\mathrm{P}=$ Fósforo.

En un caso se dispuso de las facilidades para el cálculo de peso seco según método Azul de Evans (caso 1). En el resto de las pacientes se aumentó el peso seco en $500 \mathrm{~g}$ cada 2 semanas por protocolo. No se registraron hipercalcemias, a pesar de las sesiones frecuentes de HD. El promedio de calcemia fue de $8,5 \mathrm{mg} / \mathrm{dL}$. Sólo en el caso 1 , ocurrido en el año 1988, se utilizó dializadores con membrana de cuprofan. En el resto de las pacientes la membrana fue de polisulfona y en ninguna de ellas se reutilizaron los filtros.

\section{Aspectos relacionados con las complicaciones asociadas}

El promedio de presión arterial sistólica (PAS) fue de $130 \mathrm{mmHg}(\mathrm{DE} \pm 13,3)$ y el de presión arterial diastólica (PAD) fue de $87 \mathrm{mmHg}$ (DE $\pm 16 \mathrm{mmHg}$ ). Tres pacientes (50\%) debieron usar antihipertensivos. Estos aspectos y los referentes a la calcemia, fosfemia y producto CaxP se detallan en la Tabla 3.

El promedio del Hto por trimestre fue as- cendente, desde $22,7 \%$ previo al embarazo hasta $30,2 \%$ en el tercer trimestre. Ochenta por ciento de los pacientes requirieron dosis iguales o superiores a $10.000 \mathrm{UI} /$ semanales de eritropoyetina (EPO). El caso 1 es especial, puesto que en ese momento no se disponía de EPO ni fierro endovenoso, por lo cual requirió múltiples transfusiones durante el curso del embarazo. El comportamiento del Hto y el tratamiento de la anemia se detallan en la Figura 1 y la Tabla 4.

El IMC promedio previo al embarazo era de $23,2(\mathrm{DE} \pm 1,4)$ y la albuminemia promedio de 2,9 $(\mathrm{DE} \pm 0,3)$. La prescripción nutricional se ajustó a cada paciente, pero se intentó que cada una de ellas lograra una ingesta de entre $30-35 \mathrm{kcal} / \mathrm{kg}$ y 1,8-2 g/proteína/kg al día. Sólo en el caso 2 (albuminemia basal 2,3 mg/dL) se indicaron $100 \mathrm{~g}$ de aminoácidos esenciales endovenosos intra diálisis el primer trimestre, debido al pobre incremento de la albúmina. El promedio de albuminemia en la muestra en el tercer trimestre fue de $3,4 \mathrm{mg} / \mathrm{dL}$ $(\mathrm{DE} \pm 0,16)$. 

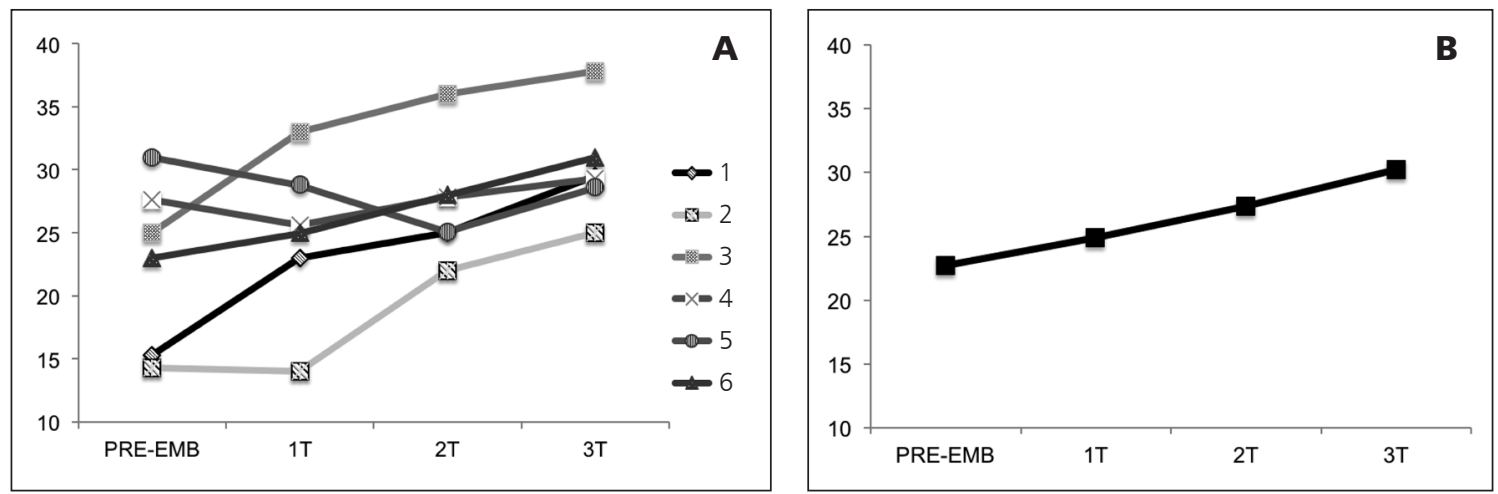

Figura 1. A. Hematocrito individual, pregestacional y su evolución por trimestre (T) de embarazo. B. Promedio del hematocrito pregestacional y evolución del promedio de los hematocritos por trimestre (T) de embarazo en seis pacientes.

Tabla 4. Tratamiento de la anemia de 6 pacientes con ERC tratadas con HD frecuente y prolongada

\begin{tabular}{|lcccccc|}
\hline & Caso 1 & Caso 2 & Caso 3 & Caso 4 & Caso 5 & Caso 6 \\
\hline Eritropoyetina (Ul/semana) & - & 12.000 & 8.000 & 12.000 & 12.000 & 10.000 \\
\hline Fierro endovenoso (mg/mes) & - & 100 & 200 & 200 & 400 & 400 \\
\hline Transfusión glóbulos rojos (Unidades) & 10 & 4 & - & - & - & - \\
\hline
\end{tabular}

\section{Aspectos obstétricos y perinatales}

El primer síntoma de embarazo fue hiperemesis en 3 de los 6 casos. Dos de las pacientes presentaron amenorrea y en una el diagnóstico se estableció a partir del estudio de distensión abdominal. El promedio de edad gestacional (EG) en el momento del diagnóstico fue de 13,4 semanas (DE $\pm 4,67)$. La totalidad de los partos fueron prematuros y por cesárea. La EG promedio al momento del parto fue de 33 semanas $( \pm 1,7)$. La causa más frecuente de interrupción del embarazo fue la colestasia intrahepática del embarazo (CIE), en 4 de los 6 casos. Se registró un caso de oligohidroamnios, uno de polihidroamnios y otro de retardo del crecimiento intrauterino (RCIU). Sesenta y seis por ciento (4/6) de los RN fueron adecuados a la edad gestacional. El promedio de Hto neonatal fue 50,3\% (DE $\pm 0,9)$ (Tabla 1$)$.

\section{Discusión}

Presentamos una serie de 6 embarazos en HD crónica en un período de 27 años. En nuestra serie 5 de los 6 embarazos ocurrieron después del año 2000. En la literatura existen 10 reportes posteriores a este año. La serie de Chao et al (2002) proporciona un número importante, con 15 mujeres y 18 embarazos ${ }^{9}$. Luders et al (2010) describen recientemente el mayor número de embarazos en diálisis provenientes de un solo centro, 52 embarazos en 20 años $(1998-2008)^{7}$. En esta última serie la tasa de éxito alcanza al $87 \%$, bastante más alta si se compara con el registro europeo, a comienzo de la década de 1980-89, que reportaba una tasa de sobrevida fetal cercana al $40 \%{ }^{5}$. Es posible afirmar, entonces, que la ocurrencia de un embarazo en diálisis es cada vez más frecuente y exitosa.

Lograr buenos resultados materno-fetales en ERC es un desafío que no se limita sólo a la población en diálisis. Recientemente, Piccoli et al mostraron que los resultados en mujeres con ERC son peores que en la población general. En la etapa 1 de la ERC más de 50\% de las embarazadas requieren un parto por cesárea, $30 \%$ tienen partos prematuros y casi $28 \%$ de los $\mathrm{RN}$ requieren cuidados neonatológicos intensivos. Este fenómeno se acentúa a medida que disminuye la función renal y en pacientes con ERC en etapas 4 y 5, 75\% de los RN son pequeños para la edad gestacional y $100 \%$ de los partos son prematuros, con un promedio de edad gestacional de 32 semanas, lo cual es bastante similar a nuestros resultados. En 
la serie de Piccoli, la proteinuria mayor a $1 \mathrm{~g}$ /día se correlacionó con el requerimiento de cuidados neonatológicos intensivos y la hipertensión arterial con el parto prematuro ${ }^{10,11}$.

Desde un punto de vista diagnóstico, $60 \%$ de las pacientes en HD tiene amenorrea secundaria a hiperprolactinemia y anemia, por lo cual la hiperemesis o el hallazgo ecográfico incidental suelen ser las vías diagnósticas más frecuentes ${ }^{12,13}$. En nuestra serie hubo 3 pacientes con hiperemesis, 2 con amenorrea y en una el diagnóstico fue ecográfico en el contexto del estudio de una distensión abdominal. El promedio de edad gestacional al diagnóstico fue de 13,4 semanas, por lo que el porcentaje de embarazos clínicamente no detectados puede ser mayor, con una alta tasa de abortos ${ }^{12}$.

¿Cómo se relaciona la dosis de diálisis con el éxito del embarazo? Luders et al hacen una distinción entre las mujeres en que la concepción ocurrió antes de iniciar HD y aquellas que ya estaban con terapia de sustitución renal. El primer grupo recibió un tratamiento menos intensivo, $12 \mathrm{~h} / \mathrm{se}$ mana, dado que tenían función renal residual. El segundo grupo recibió en promedio $15 \mathrm{~h} /$ semana. No se observaron diferencias en términos de edad y peso gestacional, porcentaje de partos prematuros ni mortalidad neonatal. En las series de Bamberg et al $(2007)^{14}$, Haase et al (2005) $)^{15}$ E Eroglu et al $(2004)^{16}$, donde las pacientes tuvieron al menos $24 \mathrm{~h}$ de diálisis a la semana y un objetivo de NUS preHD menor de $20 \mathrm{mg} / \mathrm{dL}$ no hubo mortalidad fetal ni neonatal. Barua et al $(2008)^{17}$ reportan 6 casos de embarazadas con HD nocturna que alcanzaron $48 \mathrm{~h} /$ semanales de diálisis, obteniendo 6 partos exitosos y sólo 1 antes de las 36 semanas. Hou recomienda que las pacientes alcancen $20 \mathrm{~h}$ semanales como mínimo ${ }^{18}$. En nuestra serie, el promedio de horas de diálisis semanal fue de casi 20 h y el NUS preHD de 33 mg/dL. Esto representó para las pacientes incrementar aproximadamente al doble sus horas de hemodiálisis semanales. Las ventajas de diálisis frecuentes y prolongadas son mantener una ultrafiltración suave que impide la hipotensión y la reducción del flujo placentario y alcanzar productos nitrogenados bajos, lo cual disminuiría la exposición fetal a toxinas urémicas y mejoraría la nutrición materna y fetal, permitiendo una mayor ingesta calórico proteica ${ }^{19-21}$.

Nuestros datos y los de otras series tienen cierta heterogeneidad debido a las diferencias en la etiología de la ERC, el tiempo de permanencia en diálisis y el tratamiento nutricional y de la anemia. En este sentido se hace difícil proponer conductas terapéuticas uniformes, dado que gran parte de la terapia es indicada individualmente y modificada según la evolución del embarazo. Respecto al manejo de la anemia, nuestra meta fue mantener hematocritos cercanos a 30\%, lo cual se logró sólo en 2 de 6 casos.

En nuestra muestra 50\% de las pacientes no requirieron antihipertensivos y tres casos utilizaron metildopa, hidralazina y nifedipino, como monoterapia, con buen control de las cifras tensionales. Nuevamente, atribuir este buen control a cambios en la programación dialítica puede resultar apresurado, sin embargo, un beneficio teórico del aumento de la dosis de diálisis es la optimización en el manejo del peso seco y el control de la HTA volumen dependiente. Es importante dejar en claro que de los factores asociados a mal pronóstico del embarazo en diálisis, quizás el más importante sea la preeclampsia $(\mathrm{PE})^{5,22}$. El diagnóstico de esta entidad es muy dificultoso en una mujer con ERC avanzada, excepto en casos de PE severa.

Desde un punto de vista nutricional, nosotros indicamos un régimen diario con $35 \mathrm{cal} / \mathrm{kg}$ y $1,8 \mathrm{~g} / \mathrm{kg}$ de proteínas. No hubo hiperfosfemia, probablemente en parte gracias al aumento de la dosis de diálisis. Se aportaron además vitaminas (C, tiamina, riboflavina, niacina, B6 y ácido fólico) y minerales (calcio y zinc). A pesar de que el apoyo nutricional parenteral (NP) intradialítico no ha mostrado ventajas en la sobrevida de pacientes en HD malnutridos, en la eventualidad de un embarazo sí podría tener un rol. Tuot et al $(2009)^{23}$, describen el caso de una mujer en HD con hiperemesis gravídica, que fue apoyada desde las 14 semanas con NP total recibiendo $90 \mathrm{~g}$ de proteínas, $100 \mathrm{~g}$ de dextrosa y $40 \mathrm{~g}$ de lípidos con un total de 1,5 calorías/ml aportando 1.120 calorías por cada sesión de diálisis. La paciente tenía un régimen de $24 \mathrm{~h} /$ semana de $\mathrm{HD}$, con un Kt/V por sesión de 1,46 y no desarrolló complicaciones secundarias a la NP. La albúmina se mantuvo en $3,3 \mathrm{mg} / \mathrm{dL}$ y tuvo un $\mathrm{RN}$ de $3,5 \mathrm{~kg}$ a las 36 semanas de gestación.

Tal como se reporta en la literatura, la incidencia de parto prematuro en nuestra serie fue de $100 \%$. Sin embargo, tuvimos pocos RN pequeños para la edad gestacional o con retardo del crecimiento intrauterino, lo cual difiere de lo reportado en el resto de las series. Sí tuvimos una alta tasa 
de CIE (66\%), comparada con la prevalencia registrada en la población chilena $(4,7-27,6 \%)^{24}$, para la cual no tenemos explicación. Respecto a la paciente que presentó polihidroamnios, éste se ha asociado en diálisis con la rapidez y cantidad de solutos transportados en el escenario de una presión oncótica disminuida, lo cual resultaría en un débito de agua libre hacia la cavidad amniótica. La paciente en cuestión, se presentó muy desnutrida y requirió apoyo con aminoácidos intradiálisis en las primeras etapas de su embarazo.

De nuestra serie de casos y de la revisión aquí expuesta mencionamos lo que nos parece son los elementos centrales a considerar:

1. La frecuencia de embarazo en diálisis parece haber aumentado y tiene mejores resultados.

2. La mayoría de las series publicadas maneja a sus enfermas con HD frecuentes y prolongadas, con al menos $20 \mathrm{~h}$ semanales, lo cual podría asociarse a mejores resultados materno-fetales.

3. El manejo de la anemia es difícil y la gran mayoría de las pacientes requieren altas dosis de EPO y fierro intravenoso para cumplir objetivos mínimos de un Hto sobre 30\%.

4. La PE es el factor más asociado a peor pronóstico en el embarazo en HD. Se relaciona con mortalidad fetal y parto prematuro. El diagnóstico de PE es difícil y los esfuerzos deben estar concentrados en mantener cifras tensionales controladas.

5. Es importante mantener buenos aportes nutricionales sumando 300 Kilocalorías por la gravidez a las 1,3 calorías/kg de base, y aportes proteicos de a lo menos $1,5 \mathrm{~g} /$ proteínas $/ \mathrm{kg}$. El rol de la NP intradiálisis no está definido, aunque su uso en un reporte aislado parece auspicioso.

6. El embarazo en diálisis tiene un alto riesgo de parto prematuro. Otra complicación a considerar es el RCIU. Particularmente en nuestra serie la CIE fue la complicación más frecuente.

7. Finalmente, pensamos que para el éxito de los embarazos de nuestra serie en particular, fueron fundamentales dos aspectos: a) La sólida integración conseguida por un equipo multiprofesional de obstetras, nefrólogos, enfermeras y nutricionistas y b) El firme compromiso del equipo tratante de enfrentar los desafíos y sacar la situación adelante, poniendo especial énfasis en el cuidado riguroso de cada uno de los detalles del manejo clínico.

\section{Referencias}

1. Confortini P, Galanti G, Ancona G, Giongo A, Bruschi E, Lorenzini E. Full term pregnancy and successful delivery in patient on chronic haemodialysis. Proc Eur Dial Transplant Assoc 1971; 8: 74-80.

2. Holley JL, Reddy SS. Pregnancy in dialysis patients: a review of outcomes, complications, and management Semin Dial 2003; 16: 384-8.

3. Hou S. Frequency and outcome of pregnancy in women on dialysis. Am J Kidney Dis 1994; 23: 60-3.

4. Okundaye I, Abrinko P, Hou S. Registry of pregnancy in dialysis patients. Am J Kidney Dis 1998; 31: 766-73.

5. Registration Committee of the European Dialysis and Transplant Association. Successful pregnancies in women treated with dialysis and transplantation. $\mathrm{Br} \mathrm{J}$ Obstet Gynecol 1980; 87: 839-45.

6. Nageotte MP, Grundy HO. Pregnancy outcome in women requiring chronic haemodialysis. Obstet Gynecol 1988; 72: 456-9.

7. Luders C, Castro MC, Titan SM, De Castro I, Elias RM, Abensur $\mathrm{H}$, et al. Obstetric outcome in pregnant women on long term dialysis: a case series. Am J Kidney Dis 2010; 56 (1): 36-85.

8. Daugirdas JT, Schneditz D. Overestimation of hemodialysis dose depends on dialysis efficiency by regional blood flow and conventional two-pool urea kinetic analyses. ASAIO 1995; 41: 719-24.

9. Chao AS, Huang JI, Lien R, Kung FT, Chen PJ. Pregnancy in women who undergo long term hemodialysis. Am J Obstet Gynecol 2002; 187: 152-6.

10. Piccoli GB, Attini R, Vasario E, Conijn A, Biolcati M, D'Amico F, et al. Pregnancy and Chronic kidney disease: a challenge in all CKD stages. Clin J Am Soc Nephrol 2010; 5 (5); 844-55.

11. Piccoli GB, Conijn A, Consiglio V, Vasario E, Attini R, Deagostini MC, et al. Pregnancy in dialysis patients: is the evidence strong enough to lead us to change our counseling policy? Clin J Am Soc Nephrol 2010; 5 (1): 62-71.

12. Levy DP, Giatras I, Jungers P. Pregnancy and end-stage renal disease-past experience and new insights. Nephrol Dial Transplant 1998; 13 (12): 3005-7.

13. Al-Saran K, Sabry A. Pregnancy in dialysis patients: a case series. J Med Case Rep 2008; 2: 10-14.

14. Bamberg C, Diekmann F, Haase M, Budde K, Hocher B, Halle $\mathrm{H}$, et al. Pregnancy on intensified hemodialysis: fetal surveillance and perinatal outcome. Fetal Diagn Ther 2007; 22: 289-93.

15. Haase M, Morgera S, Bamberg C, Halle H, Martini S, Hocher B, et al. A systematic approach to managing 
Embarazos exitosos en hemodiálisis crónica - F. Espinoza et al

pregnant dialysis patients-the importance of an intensified haemodiafiltration protocol. Nephrol Dial Transplant 2005; 20 (11): 2537-42.

16. Eroğlu D, Lembet A, Ozdemir FN, Ergin T, Kazanci F, Kuşcu E, et al. Pregnancy during hemodialysis: perinatal outcome in our cases. Transplant Proc 2004; 36 (1): 53-5.

17. Barua M, Hladunewick M, Keunen J, et al. Successful pregnancies on nocturnal home hemodialysis. Clin J Am Soc Nephol 2008; 3 (2): 392-6.

18. Hou S. Pregnancy in women treated with dialysis: lessons from a large series over 20 years. Am J Kidney Dis 2010; 56: 5-6.

19. Hou S. Pregnancy in chronic renal Insufficiency and end stage renal disease. Am J Kidney Dis 1999; 33 (2): 23552 .
20. Romao JE, Luder SC, Kahhle S, Pascoal IJF, Abensur $\mathrm{H}$, Sabaga E. Pregnancy in women on chronic dialysis. Nephron 1998; 78: 410-22.

21. Galland R, Traeger J, Arkouche W, Delawari E, Fouque D. Short daily hemodialysis and nutritional status. Am J Kidney Dis 2001; 37 (S2): 95-8.

22. Podymow T, August P, Akbari A. Management of renal disease in pregnancy. Obstet Gynecol Clin North Am 2010; 37 (2): 195-210

23. Tuot S, Gibson S, Caughey A, Frasetto L. Intradialytic hyperalimentation as adjuvant support in pregnant hemodialysis patients: case report and review of the literature. Int Urol Nephrol 2010; 42: 233-7.

24. Geenes V, Williamson C. Intrahepatic cholestasis of pregnancy. World J Gastroenterol 2009; 15: 2049-66. 